\title{
Genetic Variation in Drug Transporters in Ethnic Populations
}

\author{
Cheryl D. Cropp ${ }^{1}$, Sook Wah Yee ${ }^{1}$, and Kathleen M. Giacomini ${ }^{1}$ \\ ${ }^{1}$ Department of Biopharmaceutical Sciences, University of California, San Francisco, San \\ Francisco, California, USA
}

\begin{abstract}
Drug-metabolizing enzymes and membrane transporters work in concert to play crucial roles in drug absorption, distribution, and elimination. It is well recognized that genetic variation in drugmetabolizing enzymes contributes substantially to interindividual differences in drug response. With the notable exceptions of CYP1A1 and CYP2E1, genes encoding cytochrome P450s, which are involved in the metabolism of $>80 \%$ of all drugs used in clinical practice, are highly polymorphic. ${ }^{1}$ Interethnic variation in the distribution and frequency of occurrence of variant alleles in drugmetabolizing enzymes is known to alter the rate of drug metabolism in vivo, resulting in interethnic variation in drug disposition and response.
\end{abstract}

\section{Introduction}

In contrast to our understanding of the effects of ethnicity on drug metabolism, little is known about ethnicity-related differences in the disposition and effects of drugs that are substrates of membrane transporters. Recent pharmacogenomic studies of membrane transporters describe the discovery and functional characterization of variant transporters in ethnically diverse populations. ${ }^{2}$ To our knowledge, there have been no reviews describing ethnic differences in allele frequencies, functional activities, or the clinical effects of genetic variants of membrane transporters. In this short review, we summarize findings from recent pharmacogenomic studies of transporters in two major superfamilies—adenosine triphosphate (ATP)-binding cassette and solute carrier (SLC) - that are responsible for the influx and efflux of numerous clinically important drugs. Our review focuses on transporters in the liver and kidney (see Table 1) that work together with drug-metabolizing enzymes to mediate drug absorption and disposition. In particular, we focus on ethnicity-related differences in allele frequencies and describe the functional activities of nonsynonymous variants that differ in their allele frequencies among various ethnic groups. Finally, we describe the limited studies that have directly compared drug disposition and response among ethnic groups. Clearly, there is a need for more studies examining interethnic differences in the absorption and disposition of drugs that are substrates of polymorphic transporters.

In the liver, transporters on the sinusoidal membrane of the hepatocyte (e.g., OCT1, OAT2, OATP1B1, and OATP1A2) play key roles in the hepatic uptake of drugs and thereby control access to hepatic drug-metabolizing enzymes (Figure 1a). These influx transporters also work in concert with efflux transporters on the canalicular membrane (e.g., MRP2, BSEP, BCRP, and P-gp) to excrete drugs and their metabolites into the bile (Figure 1a). Because of their critically important roles in drug absorption and disposition, identification of genetic variants in hepatic influx and efflux transporters has been the subject of many studies. Such studies have centered primarily on genetic variation in the coding region of the transporter genes, with

Correspondence: KM Giacomini, (kathy.giacomini@ucsf.edu).

Conflict of Interest: The authors declared no conflict of interest. 
a particular focus on nonsynonymous single-nucleotide polymorphisms, which have a high propensity to produce functional changes in the encoded transporter proteins. In Table 2, for each hepatic and kidney drug transporter, we show the most common nonsynonymous variant in each of three populations (African Americans, European Americans, and Asians). Frequencies of occurrence of nonsynonymous variants vary widely among the different transporter proteins. Noteworthy are the low allele frequencies of nonsynonymous variants in the organic anion transporter OAT2 $(\leq 2 \%)$, suggesting a high degree of purifying selection on the gene, SLC22A7. In contrast, OCT1 has a common nonsynonymous variant, with the allele frequency $>20 \%$ in the three populations. Also noteworthy is that there may be large interethnic differences in allele frequencies of particular transporter variants. For example, for the MRP4 variant Lys304Asn, the allele frequency ranges from 8.7\% in European Americans to 22.5\% in Asians. If this allele were associated with functional changes in MRP4, the frequency differences could result in interethnic differences in drug disposition and response.

Similarly, in the kidney, the basolateral uptake transporters (e.g., OAT1, OAT3, and OCT2) are responsible for transporting drugs from blood into renal proximal tubule cells, whereas apical efflux transporters (e.g., MRP2, MRP4, and P-gp) transport drugs into the urine for excretion (Figure 1b). In addition, the uptake transporters on the apical membrane of proximal tubules (e.g., OCTN1 and OCTN2) may reabsorb xenobiotics filtered through glomeruli back into tubule cells, whereas basolateral efflux transporters (e.g., MRP1, MRP3, and MRP6) transport these reabsorbed chemicals back into the blood. Some transporters, e.g., OCTN1 and OCTN2, have a bifunctional role, transporting selected xenobiotics in the secretory direction into the tubule lumen and particular endogenous compounds (and xenobiotics) in the reabsorptive direction.

Nonsynonymous variants of renal anionic transporters (OATs) appear to have low allele frequencies (OAT1, OAT2, OAT3, and OAT4, Figure 1b, Table 2), consistent with purifying selection on these transporters, and perhaps consistent with a low redundancy in their functions. As in the hepatocyte, ethnic differences in allele frequencies are apparent in renal transporters. For example, the most common nonsynonymous variant of the novel organic cation transporter, OCTN2, has an allele frequency of $10 \%$ in African Americans and $<1 \%$ in European Americans. For more information on these transporter variants, see PharmGKB (http://www.pharmgkb.org) and dbSNP (http://www.ncbi.nlm.nih.gov/projects/SNP/).

\section{Cellular Analysis of Variant Transporters}

Ethnic differences in allele frequencies of nonsynonymous variants in membrane transporters may not translate into ethnic differences in drug disposition or response if the particular transporter variant is not functionally significant. To understand whether such variants may be functionally significant, cellular studies characterizing the functional activity of variant transporters are important. In cellular studies, variants are constructed by site-directed mutagenesis, expressed in cellular systems, and functionally characterized. Cellular systems for studies of drug transport include stably and transiently transfected mammalian cells: human embryonic kidney-293 and Madin-Darby canine kidney cells and oocytes from Xenopus laevis. Low or absent expression of endogenous transporters and ease of transfection are two of the reasons why these cell systems are optimal for drug transport studies. A drawback of these systems is that transfected cells may not reflect the actual in vivo situation.

Typical cellular analyses of the function of transporter variants include a functional screening followed by kinetic studies of interesting variants. An example of a functional screening of nonsynonymous variants of a transporter is depicted in Figure $2 .{ }^{3}$ In this functional scan, complementary DNAs of nonsynonymous variants of the transporter OATP1A2 were constructed by site-directed mutagenesis. Then equal amounts of complementary RNA (transcribed in vitro) of the reference OATP1A2 and its 11 nonsynonymous variants were 
injected into individual oocytes from X. laevis. The extent of uptake of each of two known substrates of OATP1A2, estrone sulfate and methotrexate, was determined in the oocytes expressing OATP1A2 and its nonsynonymous variants. The results of this scan demonstrate that the Ile13Thr variant exhibited enhanced uptake of estrone sulfate and methotrexate as compared to the reference, OATP1A2. In contrast, OATP1A2-Arg168Cys, OATP1A2Gln72Asp, and OATP1A2-Asn278DEL exhibited significantly reduced uptake of estrone sulfate and methotrexate as compared to the reference, OATP1A2. As noted in Figure 2, the allelic frequency of each of the variants of OATP1A2 exhibits interethnic differences among African-American, European-American, and Asian populations. If in vitro functional studies such as the one shown in Figure 2 are translated to the in vivo situation, significant interethnic population differences in the disposition of drugs that are substrates of OATP1A2 may be observed clinically. However, additional criteria for performing clinical studies to examine interethnic differences should include prior confirmation that the transporter plays a considerable role in the drug's response and/or disposition. The mere fact that a drug is a substrate of a transporter does not necessarily imply that the transporter will be important in vivo. Diffusional processes or other transporters with redundant functions should be considered.

Urban et al. ${ }^{4}$ conducted an analysis of cellular studies in 88 protein-altering variants (including nonsynonymous variants and insertions and deletions) of 11 membrane transporters identified in DNA samples from ethnically diverse populations including African Americans, European Americans, Asians, and Mexican Americans. Twenty-two of the variants showed reduced function and, of these, 5 were shared by two or more populations, whereas 17 were unique to a single ethnic group. Most of these reduced-function variants were found at low allele frequencies; however, some were associated with allele frequencies of $>5 \%$. Assuming that reduced-function variants are associated with variation in drug disposition and response, the interpopulation differences in the allele frequencies of reduced-function variants suggest that there will be important interethnic differences in drug disposition and response to substrates of these transporters.

\section{Clinical Studies in the Pharmacogenomics of Membrane Transporters}

Clinical differences among ethnic groups in the disposition and pharmacologic effects of drugs that are substrates of transporters may be attributed to differences in genetic and environmental factors that influence all aspects of the pharmacokinetic and pharmacodynamic pathway. For example, substrates of transporters may also be substrates of various polymorphic enzymes. These complexities make it difficult to understand the effects that genetic differences in transporter activity produce on drug disposition and response. Further, in contrast to the numerous clinical studies assessing the effects produced by polymorphisms in drugmetabolizing enzymes on drug disposition, there have been few clinical studies assessing the effects produced by genetic variants of membrane transporters. Most clinical studies involving the transport of drugs have been conducted in a single ethnic group. To our knowledge, only a single clinical study has directly assessed variations between ethnic groups in the disposition and/or pharmacologic effects of a transported substrate. ${ }^{5}$

Ho et $a l .{ }^{5}$ compared the disposition of pravastatin, a substrate for OATP1B1, in European Americans and African Americans. Overall, they observed that pravastatin exposure (area under the curve and peak plasma concentration) was greater for European Americans than for African Americans. However, the observations were not explained by the presence of functional variants in OATP1B1 (i.e., 521T $>$ C, 388A $>$ G), which had been shown to have reduced function in in vitro assays. Clearly, other variants in $S L C O 1 B 1$ or other genes in the pravastatin pharmacokinetic pathway may account for the interethnic differences in response to exposure to pravastatin. 
Studies of the disposition of model substrates of transporters in single ethnic groups suggest that interethnic differences in drug disposition may be present. For example, BCRP-421C>A (Gly141Lys) is the most prevalent allele in Asians (40.80\%), and its allele frequency in Asians is much greater than in other ethnic groups (Table 2). Zhang et al. ${ }^{6}$ conducted a pharmacokinetic study of rosuvastatin, a cholesterol-lowering drug in the same class as pravastatin, in 41 healthy Chinese subjects. They reported that subjects with the BCRP genotypes 421CA and 421AA showed a higher exposure to rosuvastatin (after receiving the same dose) than did subjects homozygous for $421 \mathrm{C}$. The $421 \mathrm{C}>\mathrm{A}$ variant is also found in Europeans, although at a much lower allele frequency (Table 2). It follows, therefore, that the population effect of this variant on rosuvastatin disposition in individuals with European ancestry would be much lower. In fact, the recommended starting dose for individuals of Asian descent is lower than that for Europeans.

\section{Conclusions}

In this brief review, we show that there are ethnic differences in the allele frequencies of transporter variants that are important in drug disposition in the liver and kidney. We also show that transporter variants that exhibit effects on function in cellular assays have notable interethnic differences in allele frequencies, suggesting that these reduced-function variants may be a source of variation in drug disposition among ethnic groups. Finally, there have been few clinical studies of the effects produced by genetic polymorphisms in transporters on drug disposition and response. These studies, largely carried out in single ethnic groups, suggest that there may be ethnic differences in the disposition and pharmacologic effects of drugs that are substrates for transporters. Clearly, to understand all the sources of interethnic differences in the disposition and effects of transported drugs, studies are needed that directly compare the pharmacokinetics and pharmacodynamics of transported drugs among ethnic groups.

\section{Acknowledgments}

We acknowledge National Institutes of Health grants including GM56847 for support of C.D.C. and GM61390 for support of the Pharmacogenomics of Membrane Transporters project. We acknowledge James E.Shima for his helpful discussions and advice on this manuscript.

\section{References}

1. Ingelman-Sundberg M. Polymorphisms of cytochrome P450 and xenobiotic toxicity. Toxicology 2002:181-182. 447-452. [PubMed: 11893417]

2. Leabman MK, et al. Natural variation in human membrane transporter genes reveals evolutionary and functional constraints. Proc Natl Acad Sci USA 2003;100:5896-5901. [PubMed: 12719533]

3. Badagnani I, et al. Interaction of methotrexate with organic-anion transporting polypeptide $1 \mathrm{~A} 2$ and its genetic variants. J Pharmacol Exp Ther 2006;318:521-529. [PubMed: 16702441]

4. Urban TJ, et al. Functional genomics of membrane transporters in human populations. Genome Res 2006;16:223-230. [PubMed: 16354753]

5. Ho RH, et al. Effect of drug transporter genotypes on pravastatin disposition in European- and AfricanAmerican participants. Pharmacogenet Genomics 2007;17:647-656. [PubMed: 17622941]

6. Zhang W, et al. Role of BCRP 421C>A polymorphism on rosuvastatin pharmacokinetics in healthy Chinese males. Clin Chim Acta 2006;373:99-103. [PubMed: 16784736]

7. Jonker JW, Schinkel AH. Pharmacological and physiological functions of the polyspecific organic cation transporters: OCT1, 2, and 3 (SLC22A1-3). J Pharmacol Exp Ther 2004;308:2-9. [PubMed: 14576340]

8. Koepsell H, Lips K, Volk C. Polyspecific organic cation transporters: structure, function, physiological roles, and biopharmaceutical implications. Pharm Res 2007;24:1227-1251. [PubMed: 17473959]

9. Rizwan AN, Burckhardt G. Organic anion transporters of the SLC22 family: biopharmaceutical, physiological, and pathological roles. Pharm Res 2007;24:450-470. [PubMed: 17245646] 
10. Sekine T, Miyazaki H, Endou H. Molecular physiology of renal organic anion transporters. Am J Physiol Renal Physiol 2006;290:F251-F261. [PubMed: 16403838]

11. Enomoto A, et al. Molecular identification of a renal urate anion exchanger that regulates blood urate levels. Nature 2002;417:447-452. [PubMed: 12024214]

12. Iwanga T, Kobayashi D, Hirayama M, Maeda T, Tamai I. Involvement of uric acid transporter in increased renal clearance of the xanthine oxidase inhibitor oxypurinol induced by uricosuric agent, benzbromarone. Drug Metab Dispos 2005;33:1791-1795. [PubMed: 16135657]

13. Niemi M. Role of OATP transporters in the disposition of drugs. Pharmacogenomics 2007;8:787802. [PubMed: 18240907]

14. Tirona RG, Kim RB. Pharmacogenomics of organic anion-transporting polypeptides (OATP). Adv Drug Deliv Rev 2002;54:1343-1352. [PubMed: 12406649]

15. Ito K, Suzuki H, Horie T, Sugiyama Y. Apical/basolateral surface expression of drug transporters and its role in vectorial drug transport. Pharm Res 2005;22:1559-1577. [PubMed: 16180115]

16. Grube $\mathrm{M}$, et al. Organic anion transporting polypeptide $2 \mathrm{~B} 1$ is a high-affinity transporter for atorvastatin and is expressed in the human heart. Clin Pharmacol Ther 2006;80:607-620. [PubMed: 17178262]

17. Otsuka M, Matsumoto T, Morimoto R, Arioka S, Omote H, Moriyama Y. A human transporter protein that mediates the final excretion step for toxic organic cations. Proc Natl Acad Sci USA 2005;102:17923-17928. [PubMed: 16330770]

18. Tanihara Y, Masuda S, Sato T, Katsura T, Ogawa O, Inui KI. Substrate specificity of MATE1 and MATE2-K, human multidrug and toxin extrusions/H+-organic cation antiporters. Biochem Pharmacol 2007;74:359-371. [PubMed: 17509534]

19. Masuda S, et al. Identification and functional characterization of a new human kidney-specific $\mathrm{H}+/$ organic cation antiporter, kidney-specific multidrug and toxin extrusion 2. J Am Soc Nephrol 2006;17:2127-2135. [PubMed: 16807400]

20. Cascorbi I. Role of pharmacogenetics of ATP-binding cassette transporters in the pharmacokinetics of drugs. Pharmacol Ther 2006;112:457-473. [PubMed: 16766035]

21. Huang Y. Pharmacogenetics/genomics of membrane transporters in cancer chemotherapy. Cancer Metastasis Rev 2007;26:183-201. [PubMed: 17323126]

22. Kerb R, Hoffmeyer S, Brinkmann U. ABC transporters: hereditary polymorphisms and pharmacological impact in MDR1, MRP1 and MRP2. Pharmacogenomics 2001;2:51-64. [PubMed: 11258197]

23. Lepper ER, Nooter K, Verweij J, Acharya MR, Figg WD, Sparreboom A. Mechanisms of resistance to anticancer drugs: the role of the polymorphic ABC transporters ABCB1 and ABCG2.

Pharmacogenomics 2005;6:115-138. [PubMed: 15882131]

24. Stieger B, Meier Y, Meier PJ. The bile salt export pump. Pflugers Arch 2007;453:611-620. [PubMed: 17051391] 
a
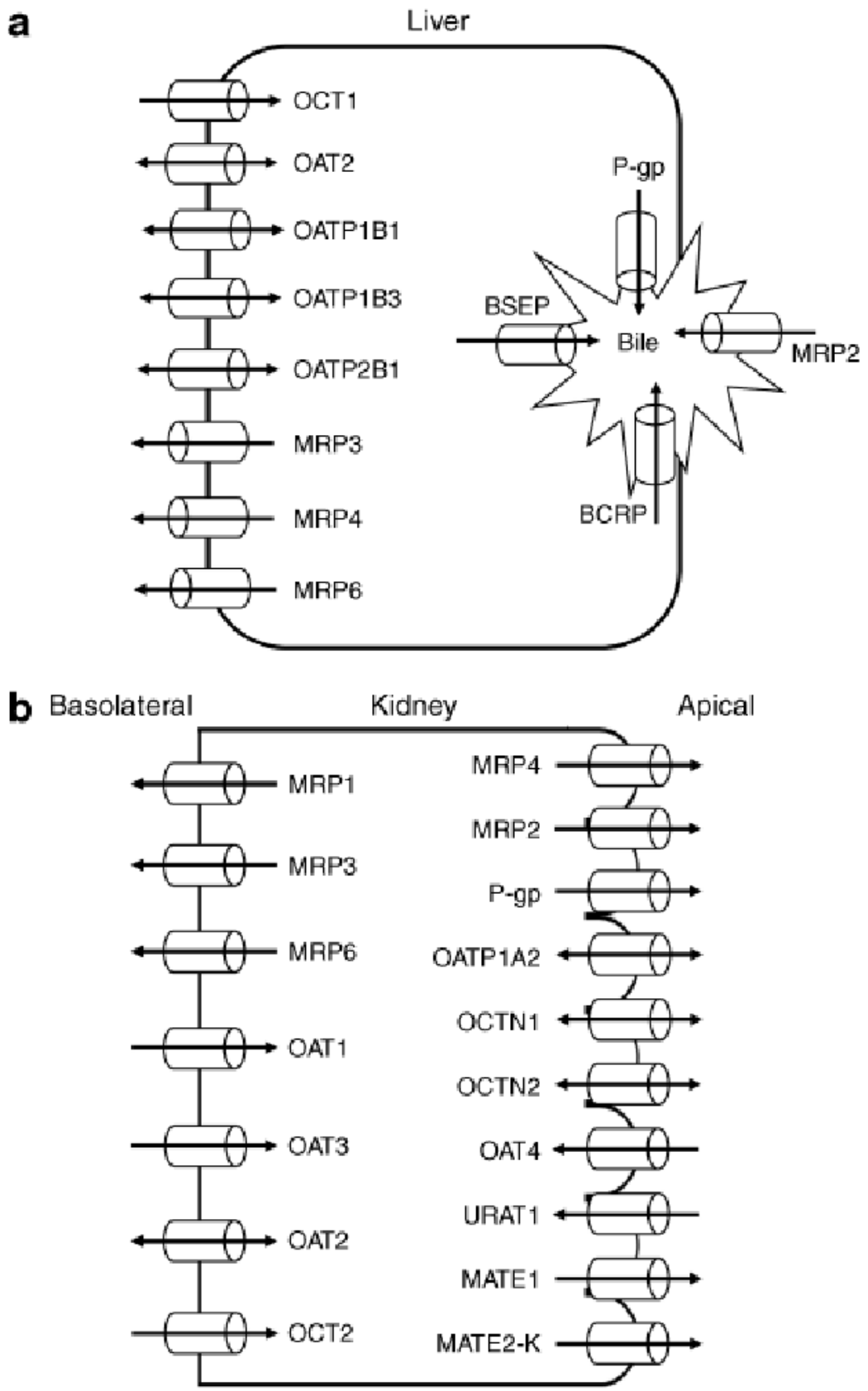

Figure 1.

Distribution of the major influx and efflux drug transporters in (a) the liver and (b) the kidney. 


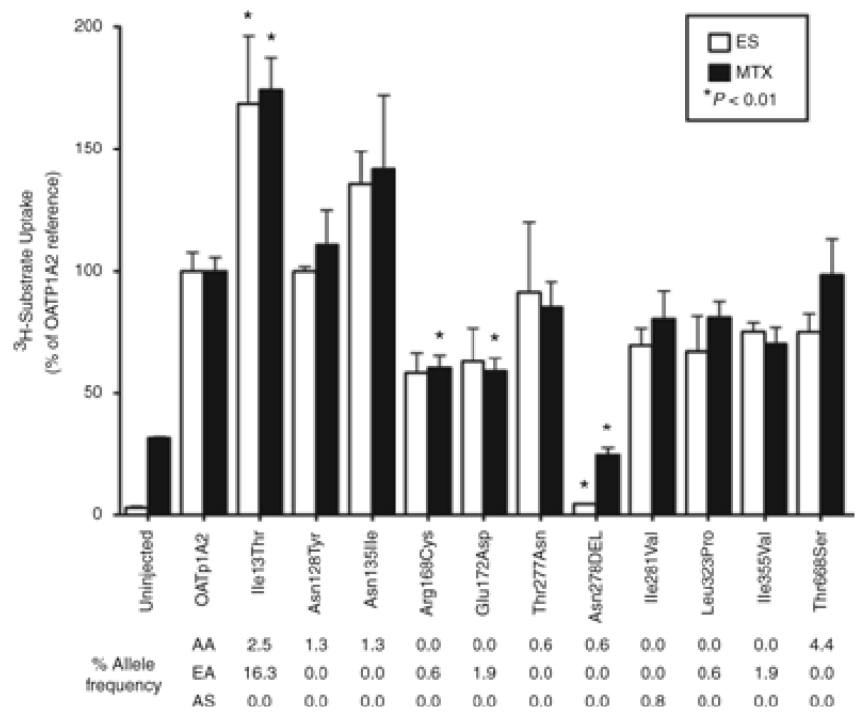

Figure 2.

Functional scan and allele frequencies of nonsynonymous variants of OATP1A2. Uptake of estrone sulfate (ES) and methotrexate (MTX) by Xenopus laevis oocytes expressing OATP1A2 and its nonsynonymous variants. The allele frequencies for African Americans (AA), European Americans (EA), and Asians (AS) are displayed for comparison. Variant positions were determined relative to the ATG start site and were based on the reference complementary DNA sequence of OATP1A2 (accession number NM_021094). 
Table 1

Human liver and kidney transporters important in drug disposition

\begin{tabular}{|c|c|c|c|c|}
\hline Gene symbol & Protein name & Full protein name & Representative substrates & References $a$ \\
\hline \multicolumn{5}{|c|}{ Influx transporters } \\
\hline$S L C 22 A 1$ & OCT1 & Organic cation transporter 1 & Metformin, oxaliplatin & 7,8 \\
\hline$S L C 22 A 2$ & OCT2 & Organic cation transporter 2 & Metformin, amantidine & 7,8 \\
\hline SLC22A4 & OCTN1 & Novel organic cation transporter 1 & Gabapentin & 8 \\
\hline SLC22A5 & OCTN2 & Novel organic cation transporter 2 & Carnitine & 8 \\
\hline SLC22A6 & OAT1 & Organic anion transporter 1 & Adefovir, tenofovir & 9,10 \\
\hline$S L C 22 A 7$ & OAT2 & Organic anion transporter 2 & Ganciclovir, allopurinol & 9,10 \\
\hline$S L C 22 A 8$ & ОАТ3 & Organic anion transporter 3 & Cimetidine, cefotaxime & 9,10 \\
\hline$S L C 22 A 11$ & OAT4 & Organic anion transporter 4 & Bumetanide, ketoprofen & 9,10 \\
\hline SLC22A12 & URAT1 & Urate anion exchanger 1 & Uric acid, oxypurinol & 11,12 \\
\hline SLCO1A2 & OATP1A2 & Organic anion transporting polypeptide A & Methotrexate, fexofenadine & 13,14 \\
\hline SLCO1B1 & OATP1B1 & Organic anion transporting polypeptide $\mathrm{C}$ & Pravastatin, repaglinide & $13-15$ \\
\hline SLCO1B3 & OАTP1B3 & Organic anion transporting polypeptide 8 & Digoxin, paclitaxel & 13,14 \\
\hline$S L C O 2 B 1$ & OATP2B1 & Organic anion transporting polypeptide B & Atorvastatin, benzylpenicillin & 13,16 \\
\hline$S L C 47 A 1$ & MATE1 & Multidrug and toxin extrusion 1 & Cimetidine, metformin & 17,18 \\
\hline$S L C 47 A 2$ & MATE2-K & Multidrug and toxin extrusion 2 & Cimetidine, metformin & 18,19 \\
\hline \multicolumn{5}{|c|}{ Efflux transporters } \\
\hline$A B C B 1$ & P-gp & P-glycoprotein & Etoposide, imantinib & $20-23$ \\
\hline$A B C B 11$ & BSEP & Bile salt export pump & Paclitaxel & $15,21,24$ \\
\hline$A B C C 1$ & MRP1 & Multidrug resistance-associated protein 1 & Methotrexate & $20-22$ \\
\hline$A B C C 2$ & MRP2 & Multidrug resistance-associated protein 2 & Doxorubicin, cisplatin & $15,20-22$ \\
\hline$A B C C 3$ & MRP3 & Multidrug resistance-associated protein 3 & Etoposide, methotrexate & 21 \\
\hline$A B C C 4$ & MRP4 & Multidrug resistance-associated protein 4 & Methotrexate & 21 \\
\hline$A B C C 6$ & MRP6 & Multidrug resistance-associated protein 6 & Anthracyclines & 21 \\
\hline$A B C G 2$ & BCRP & Breast cancer resistance protein & Mitoxantrone, doxorubicin & 20,23 \\
\hline
\end{tabular}

$\mathrm{ABC}$, adenosine triphosphate-binding cassette; SLC, solute carrier.

${ }^{a}$ Review articles describing the pharmacological, physiological, and/or pharmacogenetics of the specific membrane transporters. 
Table 2

The allele frequency of the most common non-synonymous variants for African Americans, European Americans and Asians.

\begin{tabular}{|c|c|c|c|}
\hline Protein Name & African Americans (\%) & European Americans (\%) & Asians (\%) \\
\hline \multicolumn{4}{|c|}{ Influx Transporters } \\
\hline OCT1 & Val408Met (26.5) & Val408Met (40.2) & Val408Met (23.8) \\
\hline OCT2 & Ala270Ser (11.0) & Ala270Ser (15.8) & Ala270Ser (8.6) \\
\hline OCTN1 & Thr306Ile (26.9) & Leu503Phe (41.2) & Ile306Thr (35.8) \\
\hline OCTN2 & Pro549Ser (10.0) & Val481Phe (0.6) & Phe17Leu (1.7) \\
\hline OAT1 $a$ & Arg50His (3.2) & Ile226Thr (0.6) & \\
\hline $\mathrm{OAT} 2{ }^{a}$ & Thr110Ile (2.3) & Arg227His (0.8) & \\
\hline OAT3 & Val281Ala (6.0) & Val448Ile (1.3) & Ile305Phe (3.5) \\
\hline $\mathrm{OAT}_{4} a$ & Arg121Cys (2.3) & $\operatorname{Arg} 48 \mathrm{STOP}(2.3)$ & \\
\hline URAT1 $b$ & Thr542LysfsX13 (1.5) & & Arg342His (0.8) \\
\hline $\mathrm{OATP} 1 \mathrm{~A} 2 c$ & Thr668Ser (4.4) & Ile13Thr (16.3) & Ile281Val (0.8) \\
\hline OATP1B1 & Asp130Asn (27.2) & Asn130Asp (44.1) & Asp130Asn (19.9) \\
\hline \multirow[t]{2}{*}{$\mathrm{OATP}_{1 \mathrm{~B} 3} d$} & Met233Ile (42.0) & Ala112Ser (19.0) & Ala112Ser (32.0) \\
\hline & & Ile233Met(19.0) & Ile233Met (32.0) \\
\hline OATP2B1 $e$ & Ser486Phe (40.5) & $\operatorname{Arg} 312 \mathrm{Gln}(10.8)$ & Arg312Gln (38.9) \\
\hline MATE1 $b$ & Val338Ile(5.1) & & Val480Met (0.8) \\
\hline MATE2-K ${ }^{a}$ & Pro162Leu (5.6) & Gly429Arg (0.9) & \\
\hline \multicolumn{4}{|c|}{ Efflux Transporters } \\
\hline P-gp & Ser1141Thr (11.1) & Ala893Ser (43.8) & Ala893Ser (45.0) \\
\hline BSEP & Ala444Val (47.0) & Ala444Val (42.9) & Ala444Val (33.3) \\
\hline $\operatorname{MRP}^{a}$ & Cys1047Ser (4.5) & Val353Met (0.5) & \\
\hline MRP2 & Cys1515Tyr (19.6) & Val417Ile (17.0) & Val417Ile (11.7) \\
\hline MRP3 & Pro920Ser (11.3) & Ser346Phe (2.5) & Gly11Asp (0.8) \\
\hline MRP4 & Lys304Asn (18.1) & Lys304Asn (8.7) & Lys304Asn (22.5) \\
\hline MRP6 & Val614Ala (41.2) & Val614Ala (41.9) & Val614Ala (14.2) \\
\hline BCRP & Val12Met (7.7) & Gln141Lys (8.1) & Gln141Lys (40.8) \\
\hline \multicolumn{4}{|c|}{${ }^{a}$ No non-synonymous variants in Asians. } \\
\hline \multicolumn{4}{|c|}{$\begin{array}{l}\text { c Variant positions were determined relative to the ATG start site and were based on the reference complementary DNA sequence of OATP1A2 } \\
\text { (accession number NM_021094) }\end{array}$} \\
\hline \multicolumn{4}{|l|}{$\begin{array}{l}d_{\text {Allele frequer }} \\
\text { ss } 38884356\end{array}$} \\
\hline
\end{tabular}

